LA-UR-00- $23 / 6$
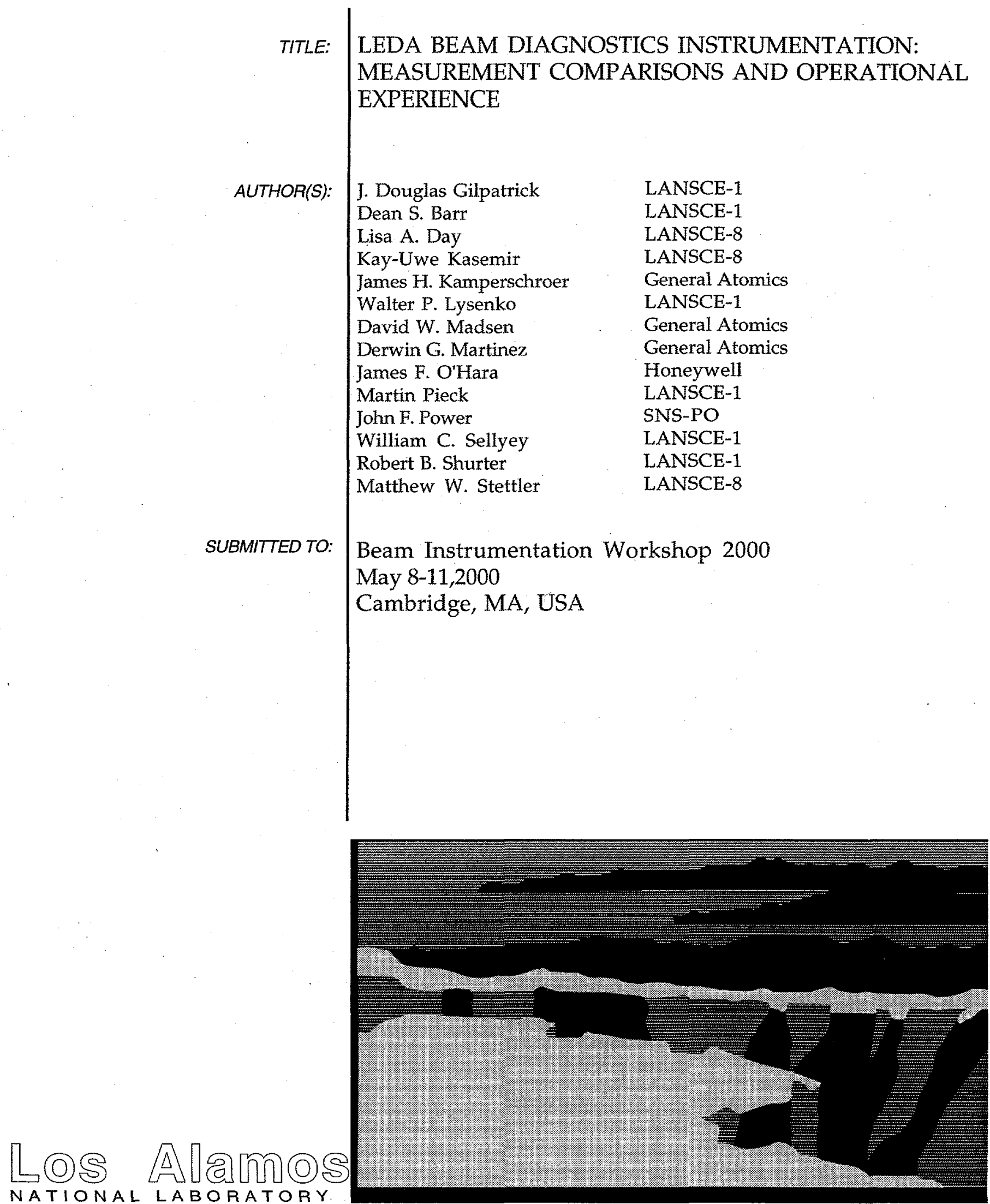

Los Alamos National Laboratory, an affirmative action/equal opportunity employer, is operated by the University of California for the U.S. Department of Energy under contract W-7405-ENG-36. By acceptance of this article, the publisher recognizes that the U.S. Government retains a nonexclusive, royalty-free license to publish or reproduce the published form of this contribution, or to allow others to do so, for U.S. Government purposes. The Los Alamos National Laboratory requests that the publisher identify this article as work performed under the auspices of the U.S. Department of Energy. 


\section{DISCLAIMER}

This report was prepared as an account of work sponsored by an agency of the United States Government. Neither the United States Government nor any agency thereof, nor any of their employees, make any warranty, express or implied, or assumes any legal liability or responsibility for the accuracy, completeness, or usefulness of any information, apparatus, product, or process disciosed, or represents that its use would not infringe privately owned rights. Reference herein to any specific commercial product, process, or service by trade name, trademark, manufacturer, or otherwise does not necessarily constitute or imply its endorsement, recommendation, or favoring by the United States Government or any agency thereof. The views and opinions of authors expressed herein do not necessarily state or reflect those of the United States Government or any agency thereof. 


\section{DISCLAIMER}

Portions of this document may be illegible in electronic image products. Images are produced from the best available original document. 


\title{
LEDA Beam Diagnostics Instrumentation: Measurement Comparisons and Operational Experience* $^{*}$
}

\author{
J. D. Gilpatrick ${ }^{*}$, D. Barr ${ }^{*}$, L. Day ${ }^{*}$, K. U. Kasemir ${ }^{*}$, J. H. \\ Kamperschroer", W. Lysenko , D. W. Madsen", D. G. Martinez", J. F. \\ O'Hara $^{\S}$, M. Pieck ${ }^{*}$, J. F. Power ${ }^{*}$, W. Sellyey ${ }^{*}$, R. B. Shurter ${ }^{*}$, M. W. \\ Stettler \\ *Los Alamos National Laboratory, Los Alamos, NM 87545, USA \\ ${ }^{\S}$ Honeywell, Albuquerque, NM, USA \\ "General Atomics, San Diego, CA 92186, USA
}

\begin{abstract}
The Low Energy Demonstration Accelerator (LEDA) facility has been used to characterize the pulsed- and cw-beam performance of a $6.7-\mathrm{MeV}, 100-\mathrm{mA}$ radio frequency quadrupole (RFQ). Diagnostic instrumentation, primarily located in a short beam transport downstream of the RFQ, allow facility commissioners and operators to measure and monitor the RFQ's accelerated and total beam transmission, beam loss, bunched beam current, beam energy and output phase, and beam position. Transverse beam profile measurements are acquired under both low and high duty-factor pulsed beam conditions using a slow wire scanner and a camera that images beam-induced fluorescence. The wire scanner is also used to acquire transverse beam emittance information using a technique known as a "quad scan". This paper reviews the measurement performance and discussés the resulting data.
\end{abstract}

\section{INSTRUMENTED BEAM TRANSPORT}

The primary purpose of the high energy beam transport (HEBT) is to safely transport the nominally $6.7-\mathrm{MeV}$ beam from the exit of the RFQ to a $670-\mathrm{kW}$ beam stop $(1,2)$. The HEBT also serves as a platform for beam instrumentation that enabled the accelerator commissioners and operators to characterize the RFQ output beam and monitor the beam during both pulsed and $\mathrm{cw}$ beam operation. This particular HEBT uses four quadrupole magnets ( $\mathrm{Q} \# 1$ through $\mathrm{Q} \# 4$ as pictured in Figure 1) to transport and expand the beam sufficiently on the beam stop to limit the beam's peak and average power density. Two steering magnets (SM \#1 and SM \#2 as pictured in Figure 1), placed after the first and second quadrupole magnets, keep the beam centered throughout the HEBT. The instrumentation suite is also shown in Figure 1. In addition, a pulsed or ac current measurement was placed at the RFQ entrance so that RFQ beam transmission could be monitored during pulsed beam operation.

\footnotetext{
* This work supported by the U.S. Department of Energy.
} 
There are additional instrumentation located in the $\mathrm{H}^{+}$source and injector but since since this paper deals with the RFQ characterization and operational beam measurements,
the injector instrumentation located in a low energy beam transport will not be discussed.

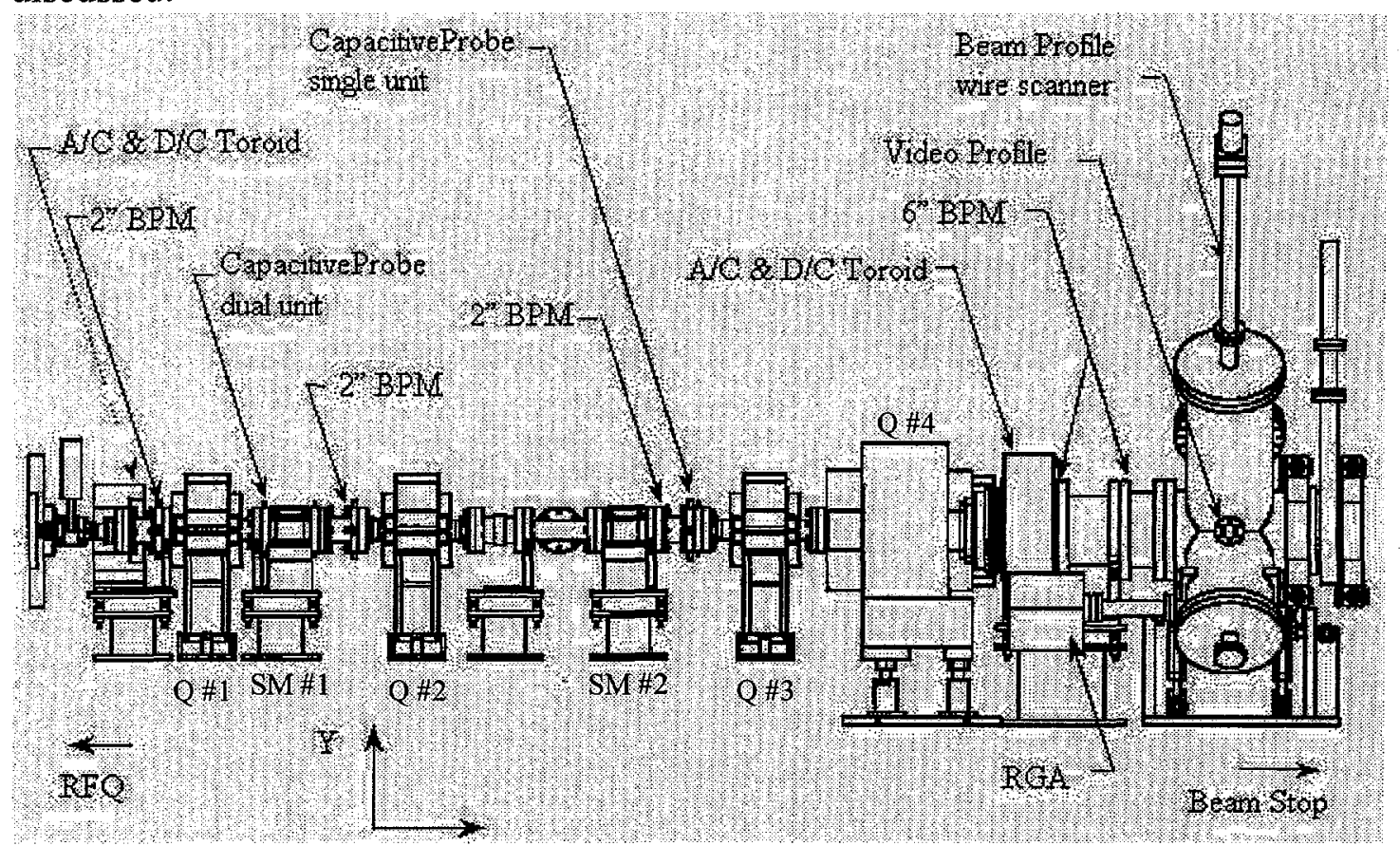

Figure 1. This drawing shows the beam instrumentation mechanical layout within the short HEBT.

\section{RFQ AND HEBT BEAM TRANSMISSION AND CURRENT}

There are three types of beam current measurements: dc, pulsed or ac, and bunched beam current. Using pulsed beam current measurements at the entrance and exit of the RFQ and HEBT, RFQ and HEBT beam transmission data are acquired during all phases of the RFQ operation $(3,4)$. Cw beam transmission was obtained in similar fashion using Bergoz ${ }^{\circledR}$ DCCTs. If the RFQ accelerating fields are set to a proper level, only particles caught within the rf bucket are both accelerated to the nominal beam energy and bunched at the RFQ resonant frequency (i.e., $350 \mathrm{MHz}$ ). Therefore, to measure the accelerated RFQ beam transmission, the RFQ output bunched beam current is compared to the total input pulsed or cw beam current. Figure 2 plots both the total and accelerated RFQ and the HEBT total beam transmission as a function of the RFQ accelerating field level set point.

Early in the RFQ operation, both the RFQ cw and pulsed beam transmission measurments displayed transmission efficiencies of greater than $100 \%$, clearly indicating a faulty measurement. The suspected cause of this faulty condition was forward-scattered electrons passing through the RFQ entrance toroid. The problem was solved by the addition of an electron trap in the RFQ entrance end wall. The ringshaped trap's primary purpose was to maintain the background neutralization gas pressure in the beam region near the RFQ entrance so that the beam's divergence 
angle could be optimized. However, an additional benefit of disallowing forward streaming electrons resulted in an accurate (i.e., typically $<1 \%$ ) pulsed-beam RFQ transmission measurement.

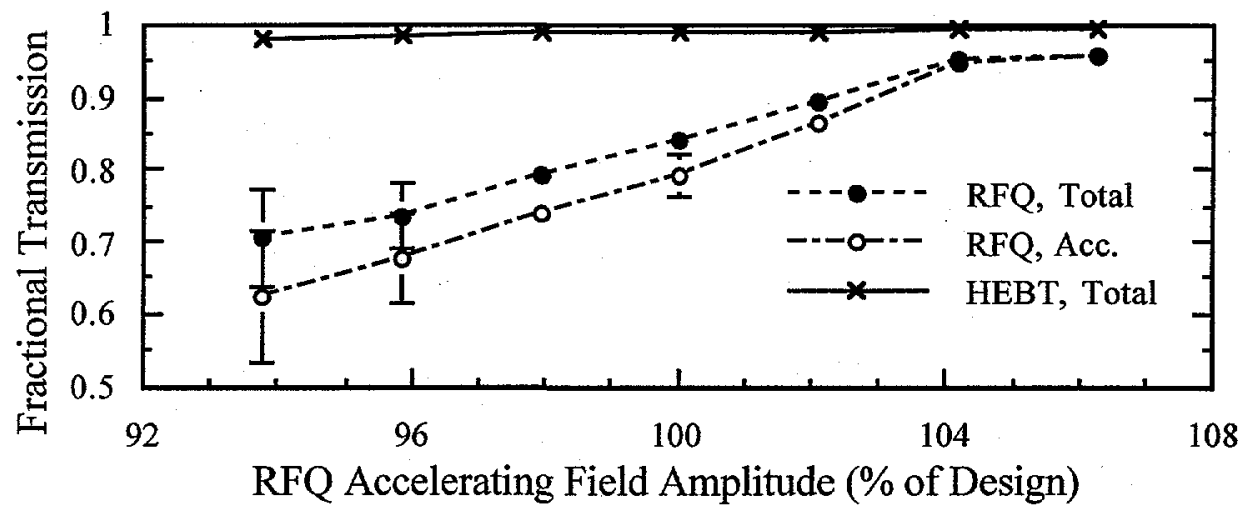

FIGURE 2. These data describe the RFQ total and accelerated and the HEBT total beam transmission as a function of the RFQ accelerating field amplitude. Vertical error bars describe the transmission variations due to beam current variations. These data were acquired under low duty-factor pulsed beam operation sampled in the last $50-\mu \mathrm{s}$.

Due to the ionizing radiation levels in the beam line near the Bergoz DCCTs, the supplied analog electronics were installed outside the beam tunnel. This decision resulted in an addition of approximately $50 \mathrm{~m}$ of a cable between the multi-core toroidal DCCT and its associated electronics. To compensate for these errors, the DCCT electronics were readjusted and the bandwidth was reduced to $100 \mathrm{~Hz}$. Unfortunately, the resulting adjustments and additional cable resulted in a 1-mA drift. During the next few months, this drift will be corrected.

\section{BEAM LOSS}

There are two types of beam loss measurements: a differential current measurement using two sequential pulsed beam current measurements, and the measurement of the ionizing radiation using both ionization chambers and proportional counters. Within each pulsed-current monitor's processor resides a digital-signal-processor (DSP). This DSP allows the processors to apply an in-situ calibrator correction to the current measurement signal on every 2 - $\mu$ s digitized sample $(3,4)$. These sampled signals are then digitally integrated and compared to a predefined lost beam-charge condition. If too much lost charge is detected as defined by the operator screen input, a digital logic signal line instructs the fast protection system to shut off the injected beam. This differential lost-current VXI-module hardware performed reliably even though there were occasional crate controller and Ethernet faults.

Ionization chambers detect prompt ionizing radiation resulting from the interaction between the proton beam and beam line components (5). For $6.7-\mathrm{MeV}$ protons, the amount of ionizing radiation is typically less than what much higher energy beams would produce. To verify that the ionization chambers were sufficiently sensitive to 
lost beam currents of less than $0.1 \mathrm{~mA}$, a test was configured using the slow wire scanner. The wire scanner's three $0.1-\mathrm{mm} \mathrm{SiC/C}$ fibers were inserted into the core of the beam distribution. Under these conditions, approximately $0.9 \mathrm{~mA}$ of beam current intercept the fiber. Figure 3 shows the indicated lost beam current. The result shows the standard deviation in the indicated lost current to be approximately $0.06-$ and $0.09-$ $\mathrm{mA}$ when the wire is in and out of the beam, respectively. This would imply a detected lost-current precision of approximately $0.1 \mathrm{~mA}$. Of course, this precision will be different for protons on stainless steel beam pipe.

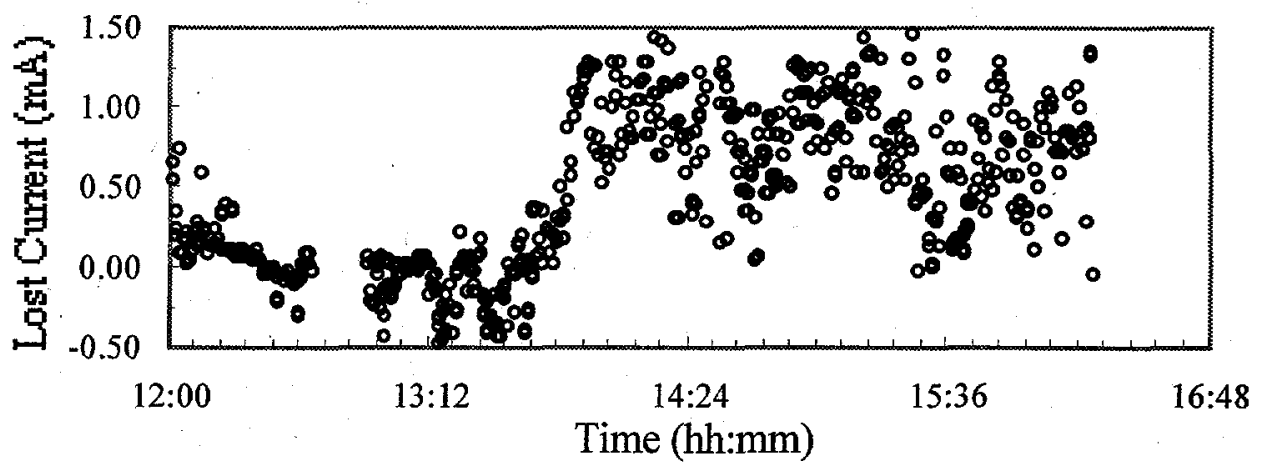

Figure 3. The lost beam current as a function of time as detected by the ionization chamber nearest the slow wire scanner. The wire scanner's three $\mathrm{SiC}$ fibers were inserted into the beam resulting in approximately $0.9 \mathrm{~mA}$ of lost beam. With a gain and offset calibration applied, the precision of this lost beam measurement is approximately $0.1 \mathrm{~mA}$.

\section{CENTRAL BEAM ENERGY AND PHASE}

Three capacitive probes and a B-dot RFQ field probe provided the rf signals to measure the beam central energy and phase $(6,7)$. A time-of-flight technique is used to measure beam energy employing two capacitive probes separated by a known distance and differential phase measurement hardware. The output phase is measured by detecting the phase difference between the first HEBT capacitive probe and the RFQ B-dot field probe, and subtracting off the phase errors due to changes in beam energy. Figure 4 depicts the changes in beam energy and phase as they are plotted in the longitudinal phase space. The RFQ accelerating field amplitude was varied and the central energy and phase data were acquired and plotted.

Initially, it was thought that all cable errors, processor errors, etc. were subtracted off these measurements. However, it was found during the debugging of the phase measurement that the processor added up to a 5-deg absolute error in the measurement. This is equivalent to approximately $14-\mathrm{keV}$ of absolute energy error and could account for the negative offset in absolute beam energy. This error is presently being diagnosed and corrected (6). For the RFQ characterization and operation, this absolute error was sufficiently small to perform all of the required measurements. 


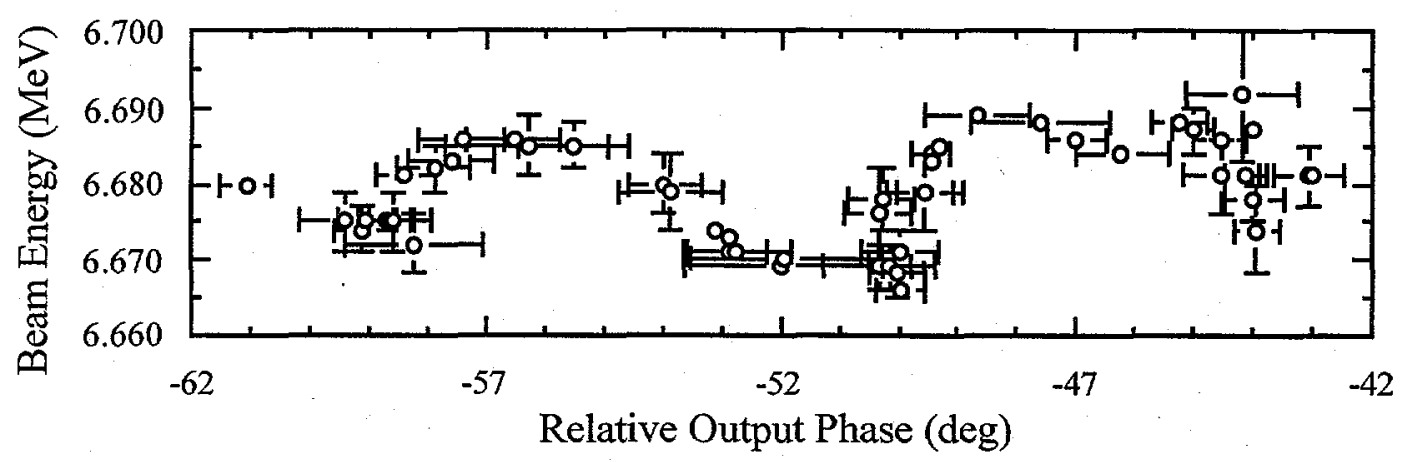

Figure 4. Longitudinal phase space with the RFQ's output beam central energy and phase plotted as a function of the RFQ accelerating field amplitude.

\section{TRANSVERSE BEAM PROFILES}

The LEDA HEBT has two methods to acquire transverse beam distributions: a slow wire scanner during low duty-factor pulsed-beam operation and a background gas fluorescence technique for high duty factor or $\mathrm{cw}$ beam operation. Both measurements are physically mounted on the HEBT such that they can view the same beam region.

The wire scanner's primary purpose is to verify that the beam width is sufficiently expanded under low duty factor pulsed conditions to guarantee beam stop safety under $\mathrm{cw}$ beam operational conditions (8). The beam size was determined by first comparing the beam's rms width and second moment with the expected size as predicted by the envelope transport code, TRACE3D. The beam expansion "rate" was inferred by changing the final quadrupole magnet's fields and calculating the rate expansion from the beam width changes. Nominally, the beam rms width at the wire scanner for the nominal HEBT tune is approximately $12 \mathrm{~mm}$ in both the vertical and horizontal planes and commonly is expanded to a $44-\mathrm{mm}$ rms width at the end of the beam stop.

With the proper transport quadrupole magnet fields and associated profile data, a technique commonly known as a "quad scan" can be performed to determine the beam's rms emittance and associated Courant-Snyder parameters. This quad scan technique was used to characterize the RFQ output beam by acquiring a series of transverse profiles for a series of upstream quadrupole magnet fields. For example, as the magnet field is reduced in the quadrupole magnet nearest the RFQ exit, a waist in the vertical plane can be moved through the plane of the wire scanner fiber. The resulting beam profile data sets can then be fit to the model of the expected beam trajectory as predicted by TRACE3D (9). Typically, this technique has been used with beams that have relatively low space charge forces such that the fit model could be approximated with a quadratic equation. However, the LEDA beam has high space charge forces, i.e., rms widths of 1- to $2-\mathrm{mm}$ and a beam current of $100-\mathrm{mA}$, so the simple models will not accurately reflect the rms emittance. Figure 5 shows the last of the series of profiles for a vertical quad scan and how the axis in which the scan is performed can have irregular shaped profiles. A new application of the quad scan 
technique presently being explored is the utilization of particle simulation codes including the appropriate space charge subroutines included as the fit model. A paper to be presented in the upcoming LINAC2000 conference will provide further details of this technique (10).

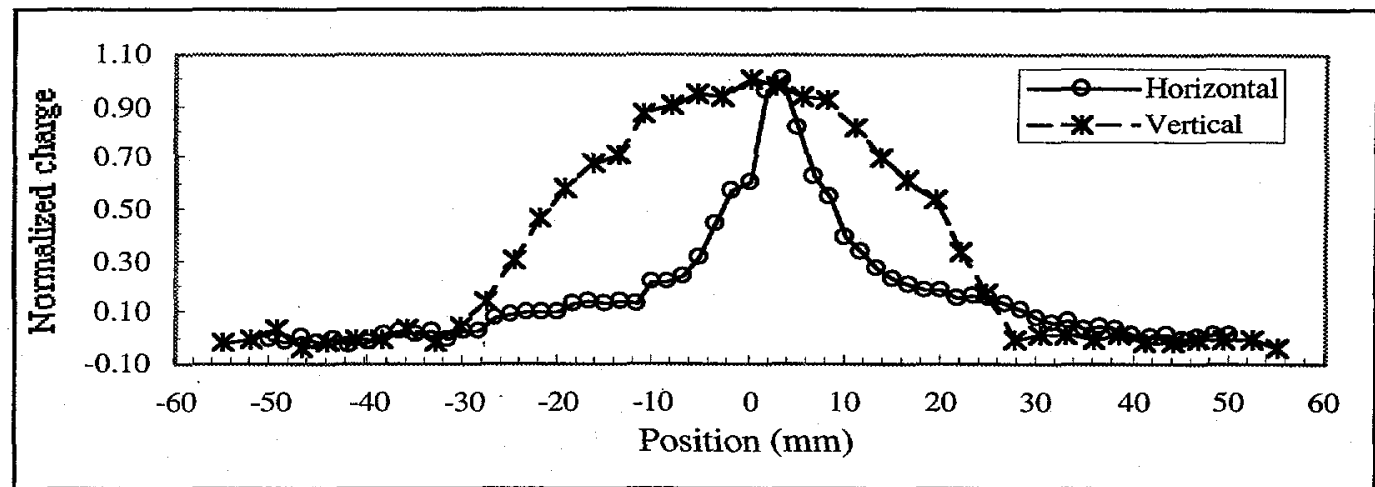

Figure 5. These particular beam distributions were taken as one of the data sets in a "quadrupole-scan" emittance measurement. These data were acquired with a $100-\mathrm{mA}, 0.08 \%$ duty factor beam.

Several experiments were conducted to understand the operational limits of the wire scanner $0.1-\mathrm{mm}$ SiC-coated carbon fiber. The wire scanner fiber was inserted into the proton beam core. Starting with a very short macropulse, the macropulse length was extended until the fiber's emitted electron current was nonlinear with time. Extending the macropulse longer eventually severed the fiber at several times the expected pulse length. Figure 6 plots the emitted electron current as a function of macropulse length. The secondary-electron (S. E.) emission region is modeled by the linear flat portion of the data, and therefore, independent of temperature. The S. E. emission current was measured to be approximately $9 \mu \mathrm{A}$ resulting in a S.E. coefficient of $3.3 \%$. What is thought to be the nonlinear thermionic emission (T.E.) region later in the macropulse is modeled by a second-order polynomial - the Richardson-Dushman equation that describes T.E. emission has a temperature-squared term. While acquiring the emitted electron data, optical spectrum data were also acquired. These data indicate agreement with the beam/fiber thermal model (i.e., radiative cooling only) up to a temperature of $1800 \mathrm{~K}$, the temperature at which the fiber transitions from the linear S.E. region to the non-linear T.E. region. Substituting this temperature into the Richardson-Dushman equation, the work function for this particular $\mathrm{SiC}$ fiber was calculated to be $3.9 \mathrm{eV}$.

The background gas fluorescence technique has been used to acquire high peak current beam profile measurements at several facilities. As a proton beam passes near background gas molecules, the proton beam excites of the molecules' electrons to a higher energy level. There is some debate whether the resulting transition to the ground state is either a molecular or atomic process (although some of the literature suggest that these lines are based on a molecular process), but in either case, the fast single transitions produce fluorescence spectral lines. Figure 7 shows the two primary $\mathrm{N}_{2}$ spectral line regions of 391- and 426-nm, respectively. If the lines are atomic, their lifetimes are on the order of $0.2-$ and $0.1-\mu \mathrm{s}$, respectively, and can be shown to be very 
short with respect to the movement of the gas molecules. Further detail of this measurement can be found in an accompanying paper (11).

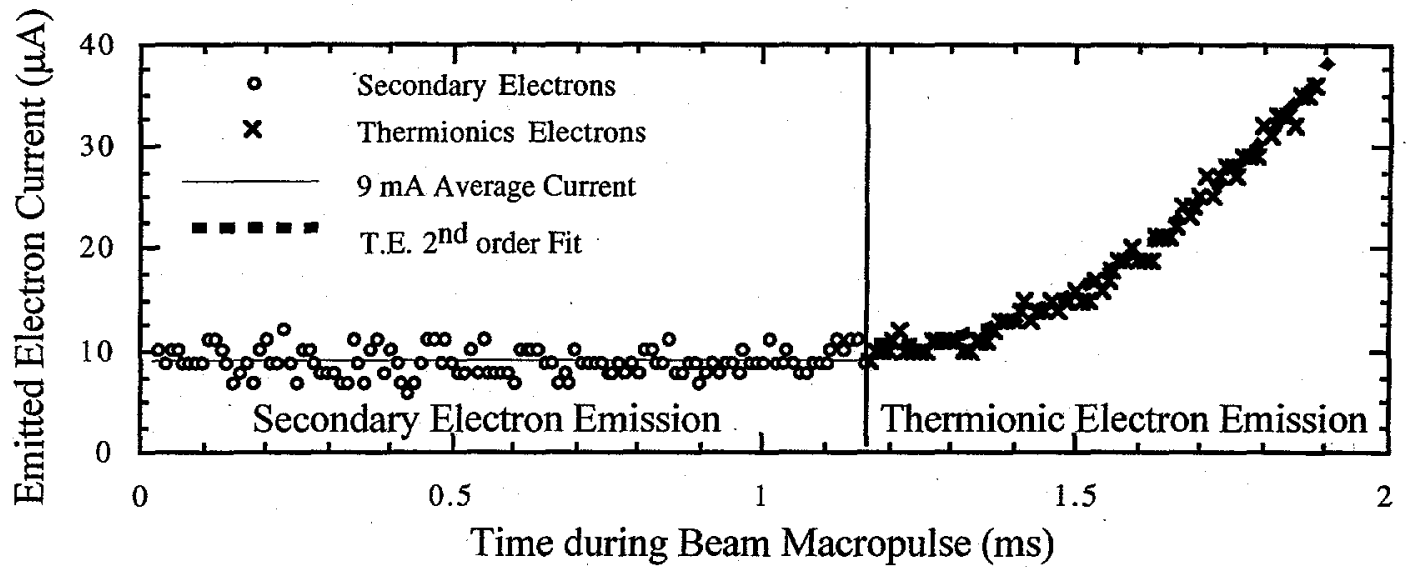

Figure 6. As beam intercepts the SiC-coated carbon fiber, the fiber emits electrons. Secondary electrons dominate in the initial part of the macropulse and thermionic electrons dominate in the later part of the macropulse. To accurately acquire a beam profile whose distribution is not distorted, the wire scanner must be operated in the linear S.E. region.

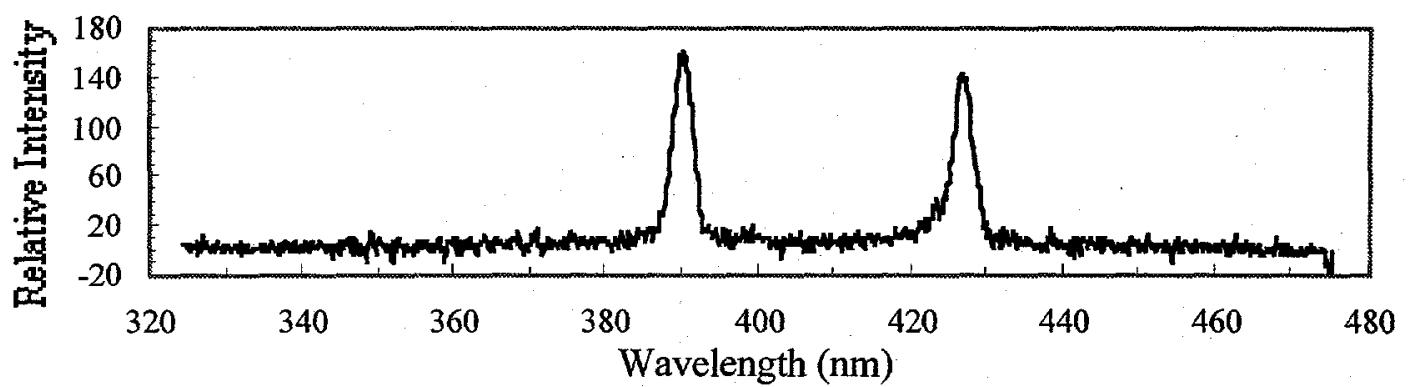

Figure 7. With $\mathrm{N}_{2}$ partial pressure of $5 \times 10^{-6}$ Torr, the two primary spectra are shown to be 391- and 426-nm, respectively.

\section{BEAM POSITION}

The beam position measurements consist of a traditional micro-stripline beam position monitor (BPM) whose downstream port is terminated in its $50-\Omega$ characteristic impedance (12). The BPM upstream port signals are fed to a log-ratio processor (13). This processor is integrated with the EPICS control system interface all within a VXI module. The VXI processor module has a built-in calibration and test function that verifies the BPM operation and error-correct calibration procedure that removes errors due to additional cable attenuation and processor log function nonconformity.

The heart of the log-ratio processor is the Analog Devices AD8307 logarithmic detector/amplifier $(14,15)$. This log amp uses the successive approximation technique to approximate the response of a true logarithmic amplifier response within a few 0.1 's $\mathrm{dB}$. To reduce these errors, an injected signal is swept through the full dynamic 
range and a correction table is generated for each of the four independent BPMelectrode channels. This error is then subtracted from each electrode at the normal sampled $200-\mathrm{kHz}$ bandwidth. The resulting accuracy of each log ratio processor axis is typically less than $+/-0.05 \mathrm{~dB}$ over greater than a $50-\mathrm{dB}$ dynamic range.

Although the beam positions were reported precisely, these indicated beam positions did not agree with the associated wire scanner indicated positions. This measurement sensitivity error was found to be errors in the BPM's sensitivity due to diffuse beam effects. It was initially felt that these errors were small (12), since measurements with previous BPMs under similar low beam energy conditions indicated that the BPM's positional sensitivity did not vary with rms beam sizevariations less than $30 \%$ of the BPM radius. However, beam tests on LEDA show that the BPM sensitivity can increase by $45 \%$ greater than the normal low- $\beta$, pencil beam calibration with rms beam width variations as little as $15 \%$ of the BPM radius.

\section{REFERENCES}

1. Gilpatrick, J. D., et al., "Low Energy Demonstration Accelerator (LEDA) Beam Instrumentation: RFQ-Accelerated Beam Results," PAC'99, New York City, NY, March 29, 1999, pp. 2214-2216.

2. Schneider, J. D., et. al., "Progress Update on the Low-Energy Demonstration Accelerator (LEDA)," PAC'99, New York City, NY, March 29, 1999, pp. 2214-2216.

3. Barr, D., et al., "LEDA Beam Diagnostics Instrumentation: Beam Current Measurement," this workshop.

4. Power, J. F., et al., "Beam Current Measurements for LEDA," PAC'99, New York City, NY, May, 1999, pp. 2241-2243.

5. Sellyey, W., et al., "Experience With Beam Loss Monitors In The Low Energy Demonstration Accelerator (LEDA)," this workshop.

6. Power, J. F., et al., "Performance of the Beam Phase Measurement System for LEDA," this workshop.

7. Gilpatrick, J. D., et al., "Experience with the Ground Test Accelerator Beam Measurement Instrumentation," in AIP Conference Proceedings 319, Santa Fe, NM, August, 1993, p. 154.

8. O'Hara, J., et al., "Slow Wire Scanner Beam Profile Measurement for LEDA," this workshop.

9. TRACE3D is a beam envelope simulation code supported by the LANL Code Group.

10.Lysenko, W., et al., "Determining Phase-Space Properties of the LEDA RFQ Output Beam", to be published in upcoming LINAC2000 Conference, Monterey, CA, August, 21-25, 2000.

11. Kamperschroer, J., "Initial Operation of the LEDA Beam-Induced Fluorescence Diagnostic," this workshop.

12. Gilpatrick, J. D., "LEDA and APT Beam Position Measurement System: Design and Initial Tests" LINAC'98, August, 1998, pp. 532-534.

13. Gilpatrick, J. D., "Comparison Of Beam-Position-Transfer Functions Using Circular BeamPosition Monitors" PAC'97, Vancouver, BC, Canada, May, 1997, pp. 2090-2092.

14. Barr, D., et al., "LEDA Beam Diagnostics Instrumentation: Beam Position Monitors," this workshop.

15. Shurter, R. B., et al., "BPM Analog Front End Electronics Based on the AD8307 Log Amplifier," this workshop. 